[15] A. L. Whiteman, The cyclotomic numbers of order ten, Proceedings of the Symposia in Applied Mathematics 10 pp. 95-111, Amer. Math. Soc., Providence, Rhode Island 1960. Ape

[16] - K. S. Williams, Explicit forms of Kummer's complementary theorems to his law of quintic reciprocity, J. Reine Angew. Math. 288 (1976), pp. 207-210.

\section{The distribution of square-free numbers}

\section{by}

R. C. Baker (Egham) and J. Pintz (Budapest)

\section{Introduction. Let}

$$
\Delta(x)=\sum_{n \leqslant x} \mu^{2}(n)-\frac{6}{\pi^{2}} x
$$

We write

$$
\psi(\theta)=\theta-[\theta]-\frac{1}{2} \quad(\theta \text { real }) .
$$

Let $\varepsilon>0$. Montgomery and Vaughan [3] showed, on the Riemann hypothesis, that

$$
\Delta(x)=-\sum_{n \leqslant M} \mu(n) \psi\left(\frac{x}{n^{2}}\right)+O\left(x^{1 / 2+\varepsilon} M^{-1 / 2}+M^{1 / 2+\varepsilon}\right),
$$

for any $M>0$. They deduced that

$$
\Delta(x)=O\left(x^{9 / 28+\varepsilon}\right) .
$$

Graham [1] improved the exponent $9 / 28$ to $8 / 25$. In the present note we sharpen this, proving

THEOREM. If the Riemann hypothesis is correct, then

$$
\Delta(x)=\dot{O}\left(x^{7 / 22+\varepsilon}\right) \text {. }
$$

The new idea is contained in Lemma 3 , which is quite similar to work of Heath-Brown ([2], Section 4). We shall make several appeals to the exponential sum estimate

$$
\sum_{a<n \leqslant b} e\left(\lambda n^{-2}\right) \ll|\lambda|^{1 / 2} a^{-1}+|\lambda|^{-1 / 2} a^{2}
$$

$\left(0<a<b \leqslant 2 a, \lambda\right.$ real non-zero). See [4], Theorem 5.9. Here $e(\theta)=e^{2 \pi i \theta}$; we also write $L=\log x$. Constants implied by ' $\ll$ ' and ' $O$ ' notations depend at most on $\varepsilon$.

Lemma 1. For some $N, H$ with

$$
x^{7 / 22}<N \leqslant x^{4 / 11}, \quad 1 / 2 \leqslant H \leqslant x^{1 / 22},
$$


we have

$$
\Delta(x) \ll L^{2}\left|\sum_{N<n \leqslant 2 N} \sum_{I I<h \leqslant 2 H} h^{-1} \mu(n) e\left(h x / n^{2}\right)\right|+x^{7 / 22+\varepsilon} .
$$

Proof. Let $J=x^{1 / 22}$. Just as in [2], Section 2, we have

$$
\psi(\theta)=-\sum_{0<|h| \leqslant J} \frac{1}{2 \pi i h} e(\theta h)+o\left(\min \left(1, \frac{1}{J|\theta| \mid}\right)\right) .
$$

Moreover,

$$
\min \left(1, \frac{1}{J\|\theta\|}\right)=\sum_{h=-\infty}^{\infty} a_{h} e(\theta h)
$$

where

$$
a_{h} \ll \min \left(L J^{-1}, J h^{-2}\right) .
$$

We apply (1) with $M=x^{4 / 11}$. By a simple splitting up argument for the interval $[1, M]$ we have

$$
\text { (6) } \Delta(x) \ll L\left|\sum_{N<n \leqslant 2 N} \mu(n) \psi\left(x / n^{2}\right)\right|+x^{7 / 22+8} \text {, }
$$

where $1 / 2 \leqslant N \leqslant M / 2$. We may evidently suppose that

$$
N>x^{7 / 22} \text {. }
$$

Now (4) gives

$$
\text { (8) } \begin{aligned}
& \sum_{N<n \leqslant 2 N} \mu(n) \psi\left(x / n^{2}\right) \\
& =-\frac{1}{2 \pi i} \sum_{0<|h| \leqslant J} \frac{1}{h} \sum_{N<n \leqslant 2 N} \mu(n) e\left(\frac{h x}{n^{2}}\right)+O\left(\sum_{N<n \leqslant 2 N} \min \left(1, \frac{1}{J\left\|x / n^{2}\right\|}\right)\right) \\
& =-\frac{1}{2 \pi i} \sum_{0<|h| \leqslant J} \frac{1}{h} \sum_{N<n \leqslant 2 N} \mu(n) e\left(\frac{h x}{n^{2}}\right)+O\left(\sum_{h=-\infty}^{\infty} a_{n} \sum_{N<n \leqslant 2 N} e\left(\frac{h x}{n^{2}}\right)\right)
\end{aligned}
$$

An application of (5) and (2) yields

$$
\text { (9) } \begin{aligned}
\sum_{h=-\infty}^{\infty} a_{h} \sum_{N<n \leqslant 2 N} e\left(\frac{h x}{n^{2}}\right) & \ll L N J^{-1}+\sum_{h=1}^{\infty} \min \left(\frac{L}{J}, \frac{J}{h^{2}}\right)\left(\frac{(h x)^{1 / 2}}{N}+\frac{N^{2}}{(h x)^{1 / 2}}\right) \\
& \ll L x^{7 / 22}+L J^{1 / 2} x^{1 / 2} N^{-1}+L J^{-1 / 2} x^{-1 / 2} N^{2} \\
& \ll L x^{7 / 22},
\end{aligned}
$$

in view of (7). The lemma follows on combining (6), (8), (9) and applying a further splitting argument to the interval $[1, J]$.
LEMMA 2. Let $1 \leqslant U \leqslant N^{1 / 3}$. For any complex function $f$ on $(N, 2 N]$, the sum

$$
\sum_{N<n \leqslant 2 N} \mu(n) f(n)
$$

may be decomposed into $O\left((\log N)^{2}\right)$ sums of the form

$$
\sum_{\substack{x<m \leqslant X_{1} \\ N<m n \leqslant 2 N}} a_{m} \sum_{\substack{Y<n \leqslant Y_{1} \\ N<m n}} f(m n)
$$

with $\left|a_{m}\right| \ll N^{\mathrm{v} / 8}, X_{1} \leqslant 2 X, Y_{1} \leqslant 2 Y$ and

$$
Y>2 N U^{-1}
$$

$$
\sum_{\substack{X<m \leqslant X_{1} \\ N<m n \leqslant 2 N}} b_{m \leqslant Y_{1}} \sum_{\substack{Y<n \\ c_{n}}} f(m n)
$$

with $\left|b_{m}\right|,\left|c_{n}\right| \ll N^{\varepsilon / 8}, X_{1} \leqslant 2 X, Y_{1} \leqslant 2 Y$ and

$$
U / 8 \leqslant Y \ll N^{1 / 2} \text {. }
$$

Proof. According to Montgomery and Vaughan [3],

$$
\sum_{N<n \leqslant 2 N} \mu(n) f(n)=S_{1}+S_{2},
$$

where

$$
\begin{aligned}
& S_{1}=-\sum_{m \leqslant U^{2} N m^{-1}} \sum_{<n \leqslant 2 N m^{-1}} a_{m} f(m n), \quad a_{m}=\sum_{\substack{d e=m \\
d, e \leqslant U}} \mu(d) \mu(e), \\
& S_{2}=-\sum_{\substack{m>U \\
N<m n \leqslant 2 N}} \sum_{\substack{n>U \\
N<m}} \mu(m) c_{n} f(m n), \quad c_{n}=\sum_{\substack{e \in n^{\prime} \\
e \leqslant U}} \mu(e) .
\end{aligned}
$$

By a splitting up argument applied to $1 \leqslant m \leqslant 2 N, 1 \leqslant n \leqslant 2 N$ we decompose $S_{1}$ and $S_{2}$ into $O\left((\log N)^{2}\right)$ nonempty subsums $S_{1, j}(j=1,2, \ldots)$, $S_{2 k}(k=1,2, \ldots)$ with domains of summation of the form

$$
X<m \leqslant X_{1}, \quad Y<n \leqslant Y_{1}, \quad N<m n \leqslant 2 N
$$

with $X_{1} \leqslant 2 X, Y_{1} \leqslant 2 Y$. Evidently $\min (X, Y)<(2 N)^{1 / 2}$. Moreover, $X \geqslant U$ and $Y \geqslant U$ in the case of sums $S_{2 k}$. Since the coefficients $\mu(m), c_{n}$ are clearly $O\left(N^{e / 8}\right)$, each sum $S_{2 k}$ is of type (II). (We may have to reverse the roles of $m$ and $n$.)

For a sum $S_{1 j}$ it may be the case that $Y>2 N U^{-1}$; in this case $S_{1 j}$ is of type (I). Suppose now that $Y \leqslant 2 N U^{-1}$, then

$$
U \leqslant 2 N Y^{-1}<8 X \leqslant 8 U^{2}
$$


also

Evidently .

$$
Y>N U^{-2} / 4 \geqslant U / 4
$$

$$
U / 8 \leqslant \min (X, Y) \ll N^{1 / 2},
$$

and $S_{1 j}$ is seen to be of type (II).

\section{Estimation of type (II) sums.}

LeMma 3. Let

$$
S=\sum_{\substack{X<m \leqslant X_{1} \\ N<m n \leqslant 2 N}} a_{m} \sum_{\substack{Y<n \leqslant Y_{1} \\ b_{n}}} b_{n<h \leqslant 2 H} c_{h} e\left(\frac{h x}{(m n)^{2}}\right),
$$

where all $a_{m}, b_{n}, c_{h}$ have modulus $\leqslant 1$. Suppose that (3) holds and that

$$
N^{2} x^{-7 / 11} H^{-1} \leqslant Y \ll N^{1 / 2} \text {. }
$$

Then

$$
S \ll H x^{7 / 22+z / 2} .
$$

Proof. Let $Q$ be a positive integer, to be specified below. Let $T_{q}$ be the set of $(n, h), Y<n \leqslant Y_{1}, H<h \leqslant 2 H$ with

$$
2 H Y^{-2}(q-1) \leqslant Q h n^{-2} \leqslant 2 H Y^{-2} q .
$$

Then

$$
S=\sum_{x<m \leqslant x_{1}} a_{m} \sum_{q=1}^{Q}\left\{\sum_{\substack{(n, h) \in T \\ N<m n \leqslant 2 N}} b_{n} c_{h} e\left(\frac{h x}{(m n)^{2}}\right)\right\} .
$$

By Cauchy's inequality,

$$
\text { (14) } \begin{aligned}
|S|^{2} & \leqslant X Q \sum_{X<m \leqslant X_{1}} \sum_{q=1}^{Q} \sum_{\substack{(n, h) \in T_{q},(r, k) \in T_{q} \\
N<m n, m r \leqslant 2 N}} b_{n} c_{n} \bar{b}_{n} \bar{c}_{r} e\left(\frac{\left(h n^{-2}-k r^{-2}\right) x}{m^{2}}\right) \\
& \leqslant X Q \sum_{n, r, h, k_{;}(15)}\left|\sum_{\substack{x<m \leqslant X_{1} \\
N<m, m r \leqslant 2 N}}^{\mid} e\left(x\left(h n^{-2}-k r^{-2}\right) m^{-2}\right)\right|
\end{aligned}
$$

Here $\sum_{n, r, h, k ;(15)}$ indicates a sum over quadruples with

$$
\begin{gathered}
Y<n, r \leqslant Y_{1}, \quad H<h, k \leqslant 2 H, \\
\left|h n^{-2}-k r^{-2}\right| \leqslant 2 H Y^{-2} Q^{-1} .
\end{gathered}
$$

The contribution to $\sum_{n, r, h, k ;(15)}$ from quadruples with $h r^{2}=k n^{2}$ is

$$
\ll X(H Y)^{1+\varepsilon / 2}
$$

by a divisor argument. The remaining quadruples can be split into $O(L)$ sets defined by (15) and

$$
\Delta / 2<\left|h n^{-2}-k r^{-2}\right| \leqslant \Delta
$$

here

$$
Y^{-4} \ll \Delta \leqslant 2 H Y^{-2} Q^{-1}
$$

Combining (14), (16) we have

$$
|S|^{2} \ll X^{2} Q(H Y)^{1+\varepsilon / 2}+L X Q \sum_{n, r, h, k ;(15),(17)}\left|\sum_{\substack{x<m \leqslant X_{1} \\ N<m n, m r \leqslant 2 N}} e\left(x\left(h n^{-2}-k r^{-2}\right) \cdot m^{-2}\right)\right|
$$

for one such $A$.

Now the number of quadruples with (15) and (17) is

$$
O\left(L^{2} H Y+\Delta H Y^{4}\right)
$$

by the argument of Heath-Brown [2] after his equation (16). We also have the bound

$$
\min \left(X,(x \Delta)^{1 / 2} X^{-1}+(x \Delta)^{-1 / 2} X^{2}\right)
$$

for the exponential sum in (14), by (2). Hence

(19) $|S|^{2} \ll X^{2} Q(H Y)^{1+8 / 2}+$

$$
\begin{gathered}
+L X Q\left(L^{2} H Y+\Delta H Y^{4}\right) \min \left(X,(x \Delta)^{1 / 2} X^{-1}+(x \Delta)^{-1 / 2} X^{2}\right) \\
\ll X^{2} Q(H Y)^{1+\varepsilon / 2}+L Q H Y^{4} x^{1 / 2} \Delta^{3 / 2}+L Q H Y^{4} x^{-1 / 2} X^{3} \Delta^{1 / 2} \\
\ll X^{2} Q(H Y)^{1+\varepsilon / 2}+L Q H Y^{4} x^{1 / 2}\left(H Y^{-2} Q^{-1}\right)^{3 / 2}+ \\
+L Q H Y^{4} x^{-1 / 2} X^{3}\left(H Y^{-2} Q^{-1}\right)^{1 / 2}
\end{gathered}
$$

in view of (18). We now set

$$
Q=\left[H N^{-2} Y x^{7 / 11}\right]
$$

note that $Q \geqslant 1$ from (12). Since $N \ll X Y \ll N$ (for $S \neq 0$ ) we have

(20) $\quad X^{2} Q(H Y)^{1+u / 2} \ll X^{2} Y^{2} N^{-2} H^{2} x^{7 / 11+8} \ll H^{2} x^{7 / 11+\varepsilon}$,

(21) $\quad L H^{3 / 2} X^{3} Y^{3} x^{-1 / 2} Q^{1 / 2} \ll L H^{2} N^{2} Y^{1 / 2} x^{-2 / 11} \ll H^{2} x^{7 / 11+\varepsilon}$.

(Here we use the upper bound in (12) together with (3).) Similarly,

$$
L Q^{-1 / 2} H^{5 / 2} Y x^{1 / 2} \ll L H^{2} N Y^{1 / 2} x^{2 / 11} \ll H^{2} x^{7 / 11+\varepsilon} \text {. }
$$

Combining (19) (22), we obtain the bound (13).

Proof of the Theorem. By Lemma 1 it suffices to show that

$$
T=\sum_{N<n \leqslant 2 N} \mu(n) \sum_{H<h \leqslant 2 H} h^{-1} e\left(\frac{h x}{n^{2}}\right) \ll x^{7 / 22+3 e / 4}
$$


whenever $N, H$ satisfy (3). We apply Lemma 2 with

$$
\begin{gathered}
f(n)=\sum_{H<h \leqslant 2 H} h^{-1} e\left(\frac{h x}{n^{2}}\right), \\
U=\max \left(1,8 N^{2} x^{-7 / 11} H^{-1}\right) .
\end{gathered}
$$

Note that $N^{5 / 3} \leqslant x^{20 / 33}$, and so $U<N^{1 / 3}$. The sum in (23) can be decomposed into $O\left(L^{2}\right)$ sums $T_{i}(i=1,2, \ldots)$ each of type (I) or type (II) in the sense of Lemma 2. Suppose $\left|T_{1}\right| \geqslant\left|T_{2}\right| \geqslant \ldots$, then

$$
|T| \ll L^{2} T_{1}
$$

Suppose for a moment that $T_{1}$ is of type (II); then

$$
T_{1} \ll N^{x / 4} H^{-1} S \text {, }
$$

where $S$ is a sum of the form that appears in Lemma 3; the condition (12) is a consequence of (11). (23) follows on combining (24), (25) and (13), and the theorem is proved in this case.

Now suppose that $T_{1}$ is of type (I). If $U=1$, then $T_{1}$ is an empty sum, so we may suppose that $U>1$. Now

$$
\begin{aligned}
T_{1} & \ll\left|\sum_{\substack{X<m \leqslant x_{1} \\
N<m n \leqslant 2 N}} a_{m} \sum_{\substack{X<n \leqslant Y_{1} \\
N<2}} e\left(\frac{h x}{(m n)^{2}}\right)\right| \\
& \ll X N^{e / 4} \max _{X<m \leqslant x_{1}} \cdot\left|\underset{\substack{X<n \leqslant Y_{1} \\
N<m n \leqslant 2 N}}{ } e\left(\frac{h x}{(m n)^{2}}\right)\right|
\end{aligned}
$$

for some $h, H<h \leqslant 2 H$. We apply (2) one last time, obtaining

$$
\begin{aligned}
T_{1} & \ll X N^{\varepsilon / 4}\left(\left(h x X^{-2}\right)^{1 / 2} Y^{-1}+\left(h x X^{-2}\right)^{-1 / 2} Y^{2}\right) \\
& \ll N^{\varepsilon / 4} h^{1 / 2} x^{1 / 2} Y^{-1}+N^{\varepsilon / 4}(h x)^{-1 / 2} N^{2} .
\end{aligned}
$$

Applying the lower bound (10) we obtain

$$
\begin{aligned}
T_{1} & \ll N^{\varepsilon / 4-1} h^{1 / 2} x^{1 / 2} U+x^{5 / 22+\varepsilon} \\
& \ll N^{\varepsilon / 4+1} x^{-3 / 22}+x^{5 / 22+\varepsilon} \ll x^{5 / 22+\varepsilon} .
\end{aligned}
$$

(23) follows on combining (24) and (26), and the proof of the theorem is complete.

\section{References}

[1] S. W. Graham, The distribution of squarefree numbers, J. London Math. Soc. (2) 24 (1981) pp. 54-64.

[2] D. R. Heath-Brown, The Pjateckil-Sapiro prime number theorem, J. Number Theory 16 (1983), pp. $242-266$.
[3] H. L. Montgomery and R. C. Vaugha n, On the distribution of squarefree numbers; in: Recent Progress in Analytic Number Theory, Academic Press, London 1981, pp. 247-256.

[4] E. C. Titchmarsh, The Theory of the Riemann Zeta Function, Oxford University Press, London 1951.

DEPARTMENT OF MATHEMATICS

ROYAL HOLLOWAY COLLEGE

EGHAM

SURREY TW20 OEX

MATHEMATICAL INSTITUTE OF THE

HUNGARIAN ACADEMY OF SCIENC

BUDAPEST, REALTANODA U. $13-15$

H-1053 HUNGARY

. 